\title{
Enquête sur les personnes ayant une maladie chronique au Canada - Composante des troubles de l'humeur et d'anxiété, 2014 : aperçu de la méthodologie
}

\author{
S. O’Donnell, M. Sc. (1); R. Cheung, Ph. D. (1); K. Bennett, Ph. D. (2); C. Lagacé, M. Sc. (1)
}

Cet article a fait l'objet d'une évaluation par les pairs.

Diffuser cet article sur Twitter

\section{Résumé}

Introduction : Il existe peu de données à propos des répercussions des troubles de l'humeur et d'anxiété sur les Canadiens et sur la gestion de ces troubles. L'Enquête sur les personnes ayant une maladie chronique au Canada - Composante des troubles de l'humeur et d'anxiété (EPMCC-THA) de 2014 a été élaborée pour combler les lacunes à ce chapitre. Le but du présent article est de décrire la méthodologie de l'EPMCC-THA de 2014 et de présenter les caractéristiques sociodémographiques de l'échantillon final.

Méthodologie : L'EPMCC-THA de 2014 est une enquête transversale de suivi menée auprès de Canadiens des 10 provinces âgés de 18 ans et plus qui souffrent d'un trouble de l'humeur ou d'anxiété diagnostiqué par un professionnel de la santé et dont on s'attend à ce qu'il dure (ou durant depuis déjà) six mois ou plus. L'enquête a été élaborée par l'Agence de la santé publique du Canada (ASPC) au moyen d'un processus itératif et consultatif avec Statistique Canada et des experts externes. Statistique Canada a mis à l'essai le contenu, a conçu la base et les stratégies d'échantillonnage et a recueilli et traité les données. L'ASPC a procédé à des analyses descriptives pour déterminer les caractéristiques sociodémographiques des répondants, a produit des estimations représentatives à l'échelle nationale à l'aide des coefficients de pondération fournis par Statistique Canada et a produit des estimations de la variance à l'aide de la méthode bootstrap.

Résultats : L'échantillon final de l'EPMCC-THA de 2014 était constitué de 3361 répondants (taux de réponse de 68,9\%). Parmi ceux qui vivaient avec un trouble de l'humeur ou d'anxiété, près des deux tiers (64\%) étaient des femmes, plus de la moitié (56\%) étaient mariés ou vivaient en union libre et $60 \%$ avaient obtenu un diplôme d'études postsecondaires. La plupart étaient jeunes ou d'âge moyen (85\%), étaient nés au Canada (88\%), étaient non Autochtones (95\%) et vivaient en milieu urbain ( $82 \%$ ). Le revenu du ménage était distribué assez également entre les quintiles de suffisance du revenu avec cependant un revenu déclaré plus souvent dans le quintile le plus bas (23\%) que dans le plus haut (17\%). Quarante-cinq pour cent ont déclaré avoir un trouble de l'humeur seulement, $24 \%$, un trouble d'anxiété seulement et $31 \%$, les deux types de trouble.

Conclusion : L'EPMCC-THA de 2014 est la seule enquête nationale auprès des ménages ayant recueilli de l'information sur les expériences des Canadiens avec un trouble de l'humeur ou d'anxiété diagnostiqué par un professionnel de la santé. Cette information permet de déterminer les secteurs où un soutien ou des interventions supplémentaires pourraient être requis et fournit un support aux futurs travaux de recherche en santé publique dans le domaine des maladies mentales.
Points saillants

- L'Enquête sur les personnes ayant une maladie chronique au Canada Composante des troubles de l'humeur et d'anxiété (EPMCC-THA) de 2014 est la seule enquête populationnelle nationale auprès des ménages qui, à ce jour, a recueilli de l'information sur les expériences des Canadiens qui vivent avec un trouble de l'humeur ou d'anxiété diagnostiqué par un professionnel de la santé.

- Au total, 3361 répondants (taux de réponse de $68,9 \%$ ) ont répondu à cette enquête, qui traitait d'un vaste éventail de sujets, dont le diagnostic, les répercussions sur les activités habituelles et les activités liées au travail, le stress, le sommeil, les relations sociales, les contacts avec des professionnels de la santé et les recommandations de ces derniers, la consommation de médicaments, les consultations psychologiques et les pratiques d'autogestion.

- L'information recueillie permet de déterminer les secteurs dans lesquels un soutien ou des interventions supplémentaires pourraient être requis. Les conclusions de l'enquête constituent aussi un support pour les futurs travaux de recherche en santé publique dans le domaine des maladies mentales.

Mots-clés : troubles de l'humeur, troubles d'anxiété, répercussions sur la santé mentale, gestion des maladies, méthodologie d'enquête, enquêtes en santé, surveillance des populations, Enquête sur les personnes ayant une maladie chronique au Canada

Rattachement des auteurs :

1. Agence de la santé publique du Canada, Ottawa (Ontario), Canada

2. Département d'épidémiologie clinique et de biostatistique, Université McMaster, Hamilton (Ontario), Canada

Correspondance : Siobhan O’Donnell, Division de la surveillance et de l'épidémiologie, Centre de prévention des maladies chroniques, Direction générale de la promotion de la santé et de la prévention des maladies chroniques, Agence de la santé publique du Canada, 785, av. Carling, Ottawa (Ontario) K1A 0K9; tél. : 613-301-7325; téléc. : 613-941-2057; courriel : siobhan.odonnell@phac-aspc.gc.ca 


\section{Introduction}

Les troubles de l'humeur et d'anxiété sont les principaux problèmes de santé mentale qui touchent les Canadiens ${ }^{1}$. En 2013, selon les estimations, 3 millions de personnes (11,6 \%) âgées de 18 ans ou plus affirmaient souffrir d'un de ces troubles ou des deux $^{2}$. Les troubles de l'humeur se caractérisent par une instabilité de l'humeur et comprennent les troubles dépressifs et les troubles bipolaires. Les troubles d'anxiété se caractérisent par un sentiment excessif et persistant d'inquiétude et de peur et comprennent le trouble d'anxiété généralisée, les phobies spécifiques, le trouble d'anxiété sociale, l'agoraphobie, le trouble obsessionnel-compulsif, le trouble panique et le trouble de stress post-traumatique ${ }^{3}$. Les troubles de l'humeur et les troubles d'anxiété sont souvent concomitants ${ }^{4-9}$ et sont également fréquemment associés à d'autres troubles mentaux (par exemple un trouble lié à l'abus de substances ou un trouble du contrôle des impulsions) ${ }^{9-11}$.

L'incapacité associée aux troubles de l'humeur et d'anxiété peut être profonde, nuire aux activités habituelles et à la capacité de conserver un emploi et perturber les relations avec les amis et les membres de la famille ${ }^{3}$. Heureusement, des soins professionnels couplés à des stratégies actives d'autogestion peuvent réduire les répercussions de ces troubles et améliorer le bienêtre des personnes qui en souffrent ${ }^{12}$. Il existe encore toutefois de nombreux obstacles à l'accès à des traitements efficaces $^{13}$. Une forte proportion de personnes aux prises avec ces troubles ne tentent pas d'obtenir des soins, et le diagnostic n'est donc pas posé. Par ailleurs, selon les observations faites à l'échelle mondiale, une forte proportion de ceux qui tentent d'obtenir des soins reçoivent un traitement sous-optimal ou n'en reçoivent aucun ${ }^{14-17}$. Le manque de connaissances, les attitudes ou croyances et la crainte de la stigmatisation en ce qui concerne les maladies mentales et leur traitement sont parmi les facteurs qui incitent le plus souvent à ne pas tenter d'obtenir des soins; les obstacles pratiques tels que le manque de temps, les coûts et l'accès aux services de santé mentale sont des motifs moins souvent évoqués ${ }^{18,19}$.

À l'heure actuelle, il manque d'information à jour sur les expériences des Canadiens qui vivent avec des troubles de l'humeur et d'anxiété, les répercussions sur leurs activités habituelles et leurs activités professionnelles et sur les méthodes de gestion de ces troubles. C'est pour combler ces lacunes que l'Agence de la santé publique du Canada (ASPC), en collaboration avec Statistique Canada, a mené l'Enquête sur les personnes ayant une maladie chronique au Canada - Composante des troubles de l'humeur et d'anxiété (EPMCC-THA) ${ }^{20}$ de 2014.

L'ASPC a commencé à élaborer l'EPMCC en 2006 en raison du manque d'information concernant certaines maladies chroniques. Les lacunes spécifiques recensées étaient l'atténuation des symptômes, l'éducation en matière de santé, l'autogestion, les soins cliniques, les interventions à visée préventive et les facteurs qui influent sur l'observance du traitement et les comportements liés à la santé. Entre 2009 et 2011, l'EPMCC a permis de recueillir des données sur l'hypertension, l'arthrite, les maladies respiratoires chroniques et le diabète et, en 2014, elle a porté sur les troubles de l'humeur et d'anxiété. Ces maladies ont été choisies en fonction de leur prévalence, de leur importance pour la santé publique et de la surveillance prioritaire exercée par l'ASPC.

À titre d'introduction à une série d'articles originaux traitant de différents aspects de la vie des Canadiens aux prises avec un trouble de l'humeur ou d'anxiété à partir des données de l'EPMCC-THA de 2014, cet article décrit les objectifs et la méthodologie de l'enquête et présente les caractéristiques de l'échantillon final.

\section{Méthodologie}

L'EPMCC-THA de 2014, une enquête transversale de suivi de l'Enquête sur la santé dans les collectivités canadiennes (ESCC) Composante annuelle de 2013, a été menée auprès de Canadiens de 18 ans ou plus atteints d'un trouble de l'humeur ou d'anxiété autodéclaré diagnostiqué par un professionnel de la santé21. L’ESCC, une enquête transversale annuelle, permet de recueillir de l'information concernant divers aspects de la santé dans la population canadienne, dont plusieurs maladies chroniques autodéclarées diagnostiquées par un professionnel de la santé22. L'ESCC peut ainsi être utilisée pour déterminer la prévalence de ces maladies chroniques au sein de la population canadienne. Elle sert aussi de base d'échantillonnage pour l'EPMCC et de source additionnelle de données sociodémographiques et de données en santé, étant donné que les réponses à l'EPMCC sont jumelées à celles de l'ESCC.

Le but principal de l'EPMCC-THA de 2014 était de fournir des renseignements détaillés sur un large éventail de sujets liés aux expériences des Canadiens atteints d'un trouble de l'humeur ou d'anxiété diagnostiqué par un professionnel de la santé, ce qui est impossible dans une enquête sur la santé en population générale telle que l'ESCC. Ainsi, l'EPMCC-THA de 2014 a permis de recueillir de l'information concernant les répercussions du trouble de santé mentale des répondants (p. ex. limitations des activités habituelles et des activités liées au travail attribuables au trouble) et les méthodes de gestion employées (médicaments, consultations psychologiques, stratégies d'autogestion, etc.). Étant donné que l'EPMCC-THA de 2014 portait uniquement sur des personnes qui avaient déclaré avoir reçu un diagnostic de trouble de l'humeur ou d'anxiété, les estimations produites reflètent les caractéristiques de cette population et non pas la prévalence de ces troubles.

\section{Objectifs de l'enquête}

Les objectifs de l'EPMCC-THA de 2014 étaient d'évaluer les répercussions des troubles de l'humeur ou d'anxiété sur les activités quotidiennes et professionnelles des Canadiens adultes, de décrire la manière dont les personnes aux prises avec un trouble de l'humeur ou d'anxiété gèrent leur trouble, de recenser les obstacles aux soins et les stratégies d'autogestion et enfin de déterminer les facteurs qui ont une influence sur l'issue de la maladie mentale.

\section{Élaboration du contenu de l'enquête}

Le contenu de l'EPMCC-THA de 2014 a été élaboré par l'ASPC et Statistique Canada au moyen d'un processus consultatif itératif, avec la participation de membres du Comité consultatif sur la surveillance de la santé mentale et de la maladie mentale de l'ASPC (le Comité consultatif) ${ }^{*}$. Le contenu a été choisi en fonction des objectifs de l'enquête, des lacunes dans les données

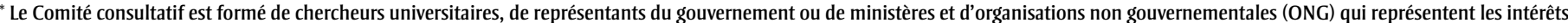
des personnes atteintes des troubles en question, telle la Société pour les troubles de l'humeur du Canada. 
repérées par l'ASPC et la Commission de la santé mentale du Canada, ainsi qu'en fonction des recommandations du Comité consultatif.

Les questions de l'enquête portant sur les domaines d'intérêt ont été tirées de diverses sources, dont des enquêtes populationnelles accessibles au public et des instruments de mesure publiés ou très connus. Certaines questions ont été modifiées après consultation auprès d'experts et d'autres relevaient de guides de pratique clinique nationaux. Les questions ont été regroupées en modules selon leur thème ou sujet. Une description de chaque module, la source des questions ou instruments et d'autres renseignements pertinents sont synthétisés dans le tableau 1.

Les questions ont été revues pour s'assurer qu'elles étaient adaptées à l'âge des répondants et à la population, qu'elles pouvaient être posées par téléphone et qu'elles répondaient aux objectifs de l'EPMCC-THA de 2014. Sur la base du questionnaire de l'ESCC 2013, les questions ont été organisées par thème, ordonnées de façon à s'enchaîner correctement, et formulées de façon que les échelles et points de vue soient normalisés et que le langage employé soit uniforme. Les questions jugées suggestives ou redondantes ont été retirées pour éviter le biais de réponse et tenir compte de la fatigue des répondants.

Le fardeau pour les répondants et les contraintes de temps associés à une entrevue téléphonique sont les principaux facteurs qui ont déterminé la longueur du questionnaire. Le contenu du questionnaire destiné à l'essai qualitatif a été élaboré en fonction de la facilité d'analyse et d'interprétation et de la possibilité de traduire l'information obtenue en messages de santé publique concrets.

Certaines questions concernant des thèmes en particulier ont été retirées avant l'essai qualitatif si elles figuraient déjà dans

TABLEAU 1

Modules du questionnaire de I'EPMCC-THA de 2014

\begin{tabular}{|c|c|c|c|c|}
\hline Module & $\begin{array}{l}\text { Thème du } \\
\text { contenu }\end{array}$ & $\begin{array}{l}\text { Nombre de } \\
\text { questions }^{\mathrm{a}}\end{array}$ & Courte description & Source \\
\hline 1. Introduction & Administratif & S.o. & $\begin{array}{l}\text { Fournit au répondant le contexte nécessaire et } \\
\text { indique le but de l'enquête. }\end{array}$ & S.o. \\
\hline $\begin{array}{l}\text { 2. État de santé } \\
\text { général }\end{array}$ & Général & 4 & $\begin{array}{l}\text { Pose aux répondants des questions au sujet de leur } \\
\text { santé générale, de la satisfaction à l'égard de leur vie, } \\
\text { de leur santé mentale et du stress dans leur vie. }\end{array}$ & $\begin{array}{l}\text { Les questions du module État de santé général de } \\
\text { l'ESCC } 2013 \text { - Composante annuelle }{ }^{23} \text { ont été } \\
\text { répétées, car elles constituent une bonne entrée } \\
\text { en la matière, elles sont sensibles aux change- } \\
\text { ments, et elles sont importantes pour évaluer } \\
\text { l'état de santé des personnes qui souffrent d'un } \\
\text { trouble de l'humeur ou d'anxiété. }\end{array}$ \\
\hline $\begin{array}{l}\text { 3. Confirmation du } \\
\text { diagnostic }\end{array}$ & $\begin{array}{l}\text { Trouble de } \\
\text { l'humeur ou } \\
\text { d'anxiété }\end{array}$ & $7(11)^{b}$ & $\begin{array}{l}\text { Confirme que le répondant appartient à la popula- } \\
\text { tion cible et pose des questions au sujet du type de } \\
\text { trouble de l'humeur ou d'anxiété, de l'âge au } \\
\text { moment du diagnostic et de l'âge au moment des } \\
\text { premiers symptômes. }\end{array}$ & $\begin{array}{l}\text { Questions adaptées du module Problèmes de } \\
\text { santé chroniques de l'ESCC } 2013 \text { - Composante } \\
\text { annuelle }{ }^{23} \text { visant à déterminer si le répondant a } \\
\text { déjà reçu un diagnostic de trouble de l'humeur ou } \\
\text { d'anxiété. Toutes les autres questions sont tirées } \\
\text { d'un cycle précédent de l'EPMCC portant sur une } \\
\text { maladie }{ }^{24} \text { et traitent des sujets d'intérêt en lien } \\
\text { avec les troubles de l'humeur ou d'anxiété. }\end{array}$ \\
\hline $\begin{array}{l}\text { 4. Consommation } \\
\text { de médicaments }\end{array}$ & $\begin{array}{l}\text { Trouble de } \\
\text { l'humeur ou } \\
\text { d'anxiété }\end{array}$ & 11 & $\begin{array}{l}\text { Traite de l'usage de médicaments sur ordonnance par } \\
\text { les répondants pour les aider à gérer leur trouble de } \\
\text { I'humeur ou d'anxiété, des raisons pour lesquelles les } \\
\text { répondants ne prennent plus ou n'ont jamais pris de } \\
\text { médicaments, du respect de la posologie des } \\
\text { médicaments et de l'utilisation de produits de santé } \\
\text { naturels. }\end{array}$ & $\begin{array}{l}\text { Questions adaptées de cycles précédents de } \\
\text { l'EPMCC portant sur des maladies précises }{ }^{24-27} \text { et } \\
\text { traitant des sujets d'intérêt en lien avec les } \\
\text { troubles de l'humeur ou d'anxiété. }\end{array}$ \\
\hline 5. Autogestion & $\begin{array}{l}\text { Trouble de } \\
\text { l'humeur ou } \\
\text { d'anxiété }\end{array}$ & $9(22)^{c}$ & $\begin{array}{l}\text { Pose aux répondants des questions au sujet des } \\
\text { mesures qu'ils ont pu adopter à la suite de leur } \\
\text { diagnostic pour gérer leur trouble de l'humeur ou } \\
\text { d'anxiété, le respect des mesures choisies au moment } \\
\text { de l'enquête, et les raisons pour ne pas s'en tenir aux } \\
\text { mesures choisies. Les questions sont similaires à } \\
\text { celles du module Recommandations cliniques afin de } \\
\text { déterminer si les répondants suivent les pratiques } \\
\text { exemplaires recommandées. }\end{array}$ & $\begin{array}{l}\text { Questions adaptées de cycles précédents de } \\
\text { I'EPMCC }{ }^{2427} \text { traitant de maladies précises et } \\
\text { basées sur les guides de pratique clinique ou les } \\
\text { pratiques exemplaires pour l'autogestion des } \\
\text { troubles de l'humeur ou d'anxiétééc,29. }\end{array}$ \\
\hline $\begin{array}{l}\text { 6. Contacts avec } \\
\text { des professionnels } \\
\text { de la santé }\end{array}$ & $\begin{array}{l}\text { Trouble de } \\
\text { l'humeur ou } \\
\text { d'anxiété }\end{array}$ & 5 & $\begin{array}{l}\text { Pose aux répondants des questions sur leurs } \\
\text { interactions avec des professionnels de la santé } \\
\text { concernant leur trouble de l'humeur ou d'anxiété au } \\
\text { cours des } 12 \text { derniers mois. Certaines questions } \\
\text { concernent les consultations psychologiques. }\end{array}$ & $\begin{array}{l}\text { Questions adaptées de l'ESCC } 2013 \text { - Composante } \\
\text { annuelle }{ }^{23} \text { et traitant des sujets d'intérêt en lien } \\
\text { avec les troubles de l'humeur ou d'anxiété. }\end{array}$ \\
\hline
\end{tabular}


TABLEAU 1 (suite)

Modules du questionnaire de l'EPMCC-THA de 2014

\begin{tabular}{|c|c|c|c|c|}
\hline Module & $\begin{array}{l}\text { Thème du } \\
\text { contenu }\end{array}$ & $\begin{array}{l}\text { Nombre de } \\
\text { questions }^{\text {a }}\end{array}$ & Courte description & Source \\
\hline $\begin{array}{l}\text { 7. Recommanda- } \\
\text { tions cliniques }\end{array}$ & $\begin{array}{l}\text { Trouble de } \\
\text { l'humeur ou } \\
\text { d'anxiété }\end{array}$ & 7 & $\begin{array}{l}\text { Traite des recommandations des médecins ou } \\
\text { d'autres professionnels de la santé pour aider les } \\
\text { répondants à gérer leur trouble de l'humeur ou } \\
\text { d'anxiété. }\end{array}$ & $\begin{array}{l}\text { Questions adaptées de cycles précédents de } \\
{\text { l'EPMCC }{ }^{2427} \text { traitant de maladies précises et }} \\
\text { basées sur les guides de pratique clinique ou les } \\
\text { pratiques exemplaires pour la gestion des troubles } \\
\text { de l'humeur ou d'anxiétééc,29. }\end{array}$ \\
\hline $\begin{array}{l}\text { 8. Limitation des } \\
\text { activités }\end{array}$ & $\begin{array}{l}\text { Trouble de } \\
\text { l'humeur ou } \\
\text { d'anxiété }\end{array}$ & 8 & $\begin{array}{l}\text { Pose aux répondants des questions au sujet des } \\
\text { limitations de leurs activités habituelles au cours des } \\
12 \text { derniers mois en raison de leur trouble de } \\
\text { l'humeur ou d'anxiété. }\end{array}$ & $\begin{array}{l}\text { Questions adaptées du module État de santé } \\
\text { général (SF-36) de l'ESCC } 2013 \text { - Composante } \\
\text { annuelle }{ }^{23} \text { et traitant des sujets d'intérêt en lien } \\
\text { avec les troubles de l'humeur ou d'anxiété. }\end{array}$ \\
\hline $\begin{array}{l}\text { 9. Limitation des } \\
\text { activités } \\
\text { professionnelles }\end{array}$ & $\begin{array}{l}\text { Trouble de } \\
\text { l'humeur ou } \\
\text { d'anxiété }\end{array}$ & 7 & $\begin{array}{l}\text { Pose aux répondants des questions au sujet de leur } \\
\text { emploi actuel et de leurs emplois passés ainsi que des } \\
\text { activités liées au travail qui ont dû être modifiées en } \\
\text { raison de leur trouble de l'humeur ou d'anxiété. }\end{array}$ & $\begin{array}{l}\text { Questions adaptées du National Health Interview } \\
\text { Survey (NHIS) des ÉtatsUnis }{ }^{30} \text {. }\end{array}$ \\
\hline 11. Stress & $\begin{array}{l}\text { Trouble de } \\
\text { l'humeur ou } \\
\text { d'anxiété }\end{array}$ & 3 & $\begin{array}{l}\text { Pose aux répondants des questions au sujet des } \\
\text { différentes sources de stress dans leur vie de tous les } \\
\text { jours, de leur capacité à y faire face et de leur } \\
\text { principale source de stress. }\end{array}$ & $\begin{array}{l}\text { Les questions du module Sources de stress de } \\
\text { I'ESCC } 2013 \text { - Composante annuelle }{ }^{23} \text { ont été } \\
\text { répétées, car elles sont sensibles aux change- } \\
\text { ments. La question sur les sources de stress a été } \\
\text { modifiée pour faire en sorte que les choix de } \\
\text { réponse soient lus par les répondants. }\end{array}$ \\
\hline $\begin{array}{l}\text { 12. Échelle de } \\
\text { provisions sociales }\end{array}$ & $\begin{array}{l}\text { Trouble de } \\
\text { l'humeur ou } \\
\text { d'anxiété }\end{array}$ & 10 & $\begin{array}{l}\text { Traite de la mesure dans laquelle les relations sociales } \\
\text { des répondants procurent divers types de soutien } \\
\text { social. }\end{array}$ & $\begin{array}{l}\text { Échelle de provisions sociales (24 éléments) } \\
\text { développée par Cutrona et Russell (1987) }{ }^{31} \text {, et vali- } \\
\text { dation de la version française par Caron (1996, } \\
2013)^{32,33} \text {. }\end{array}$ \\
\hline
\end{tabular}

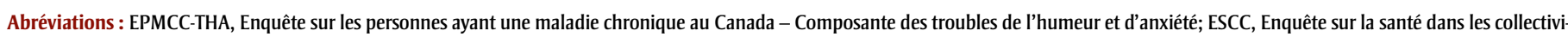
tés canadiennes; s.o., sans objet.

${ }^{\text {a }}$ Le nombre de questions posées à chaque répondant dépend du nombre de questions sautées et de la mesure dans laquelle certaines questions s'adressent aux répondants.

${ }^{\mathrm{b}}$ Bien que ce module renferme 7 questions, 2 questions sont divisées en plusieurs parties, pour un total de 11 questions.

' Bien que ce module renferme 9 questions, quelques questions sont divisées en plusieurs parties, pour un total de 22 questions.

l'ESCC 2013 et pouvaient donc être obtenues en jumelant les enregistrements (p. ex. niveau de scolarité et nutrition), si elles étaient trop vastes pour être adéquatement abordées pendant la période allouée à l'enquête (p. ex. indice de l'état de santé, stade de modification du comportement), si elles exigeaient des explications détaillées ou encore si une enquête longitudinale serait plus appropriée pour les mesurer (p. ex. rétablissement).

Des questions ont été ajoutées en vue d'obtenir l'autorisation des répondants pour le partage des données et le jumelage de leurs réponses à l'EPMCC-THA de 2014 avec celles de l'ESCC 2013. Le questionnaire anglais a été traduit en français de façon à être utilisé dans les deux langues officielles du Canada. La traduction française du questionnaire a été validée au moyen d'un examen comparatif. Les questionnaires finaux ont ensuite été intégrés à une application d'entrevue téléphonique assistée par ordinateur (CATI), assurant que l'enquête était administrée de façon uniforme par les intervieweurs.

\section{Essai qualitatif}

Le Centre d'information sur la conception de questionnaires (CICQ) de Statistique Canada a mis à l'essai les questionnaires français et anglais de l'EPMCC-THA de 2014 au moyen d'entrevues individuelles avec une sélection de répondants.

Les essais qualitatifs ont été réalisés en mars 2013 à Toronto (anglais) et à Montréal (français). L'ESCC 2012 - Composante annuelle a été utilisée comme base pour la sélection des répondants. Ont pris part à 
l'essai 26 répondants, soit une partie de ceux qui avaient déclaré souffrir d'un trouble de l'humeur ou d'anxiété diagnostiqué par un professionnel de la santé lors de l'entrevue pour l'ESCC 2012. S'appuyant sur des cadres théoriques et des méthodes basées sur la psychologie cognitive et sociale $^{34,35}$, chaque entrevue explorait les quatre étapes du processus cognitif utilisées pour répondre à un questionnaire : 1) comprendre les catégories de questions et de réponses, 2) se rappeler et/ou chercher l'information demandée, 3) réfléchir à sa réponse et décider des éléments à déclarer et 4) répondre. Toutes les entrevues ont été réalisées par un intervieweur compétent du CICQ et observées derrière une glace sans tain par des membres de l'équipe de l'EPMCC-THA de 2014 (personnel de Statistique Canada et de l'ASPC).

Le CICQ a produit des rapports sur les résultats des essais qualitatifs en anglais et en français, qui ont servi à finaliser le contenu du questionnaire. De manière générale, l'enquête a été bien accueillie par les participants. Ceux-ci ont indiqué que les modules du questionnaire s'enchaînaient bien et que les questions des modules couvraient les principaux aspects de la vie avec un trouble de l'humeur ou d'anxiété. Cependant, les participants ayant eu quelques difficultés à répondre aux questions des modules qui mesuraient les concepts de résilience et de maîtrise à cause de l'alternance de concepts positifs et de concepts négatifs et de la nature délicate des sujets couverts, l'équipe de l'EPMCC-THA de 2014 a décidé de retirer ces modules du questionnaire. De plus, l'équipe a modifié certains des modules conservés afin de préciser les éléments à déclarer et de bien identifier les répondants admissibles.

\section{Questionnaire final}

Le questionnaire final, d'une durée de 20 minutes, comprend 12 modules qui traitent de chacun des objectifs du cycle de l'EPMCC 2014. Le questionnaire complet peut être consulté sur le site Web de Statistique Canada (http://www23.statcan .gc.ca/imdb/p2SV_f.pl?Function = getSurvey \&SDDS $=5160$ ).

\section{Population cible}

La population cible de l'EPMCC-THA de 2014 était les Canadiens âgés de 18 ans ou plus au 31 décembre 2013 qui vivaient dans une résidence privée dans l'une des
10 provinces canadiennes et qui avaient répondu par l'affirmative à au moins l'une des deux questions suivantes de l'ESCC 2013 : «Rappelez-vous, nous sommes intéressés aux problèmes de santé diagnostiqués par un professionnel de la santé qui devraient durer ou qui durent depuis six mois ou plus. Êtes-vous atteint d'un trouble de l'humeur tel que la dépression, le trouble bipolaire, la manie ou la dysthymie? » ou " Êtes-vous atteint d'un trouble d'anxiété tel qu'une phobie, un trouble obsessionnel-compulsif ou un trouble panique? " Les résultats de l'étude concernent donc les Canadiens qui ont cherché à obtenir des soins pour un trouble de l'humeur ou d'anxiété et qui ont reçu un diagnostic. L'échantillon ne comprend pas ceux qui n'ont pas tenté d'obtenir de soins ou qui ont tenté sans succès d'en obtenir malgré la présence de symptômes qui répondaient aux critères diagnostiques, ce qui fait que l'applicabilité des résultats à ces individus est incertaine.

Les résidants des trois territoires, les personnes qui vivent dans des réserves autochtones ou sur des terres publiques, les personnes qui vivent en établissement, les membres à temps plein des Forces armées canadiennes et les résidants de certaines régions éloignées ont été exclus. À l'exception des trois territoires, les personnes exclues étaient les mêmes que celles prévues dans la base d'échantillonnage de l'ESCC 2013. Au total, ces exclusions représentent environ $3 \%$ de la population canadienne.

Par ailleurs, les répondants atteints d'un trouble de l'humeur ou d'anxiété vivant dans l'une des 10 provinces et qui répondaient à l'un des critères suivants ont été exclus de l'échantillon :

- absence de numéro de téléphone valide,

- réponse au questionnaire de l'ESCC par personne interposée,

- absence d'autorisation de communiquer les réponses aux partenaires de partage de données de Statistique Canada et de jumeler les réponses de l'EPMCC-THA de 2014 à celles de l'ESCC 2013.

\section{Stratégie d'échantillonnagé}

L'échantillon de l'enquête a été constitué en deux étapes : un premier échantillon à partir de l'ESCC 2013 et un second à partir de l'EPMCC-THA de 2014. L'échantillon de l'ESCC 2013 a été sélectionné au moyen de deux bases : la base aréolaire de l'Enquête sur la population active et une liste de numéros de téléphone, chacune comptant pour la moitié. La base d'échantillonnage de l'EPMCC-THA de 2014 est l'ensemble des répondants à l'ESCC 2013 satisfaisant aux critères de la population cible (voir section correspondante).

L'échantillon de l'EPMCC-THA de 2014 a été choisi de façon à produire des estimations fiables à l'échelle nationale par groupe d'âge et sexe. Les groupes d'âge étaient les 18 à 34 ans, les 35 à 49 ans, les 50 à 64 ans et les 65 ans et plus (tableau 2). L'échantillon a également permis de produire des estimations à l'échelle régionale (provinces de l'Atlantique, Québec, Ontario, Prairies et Colombie-Britannique). La taille de l'échantillon a été limitée par le nombre de personnes ayant déclaré lors de l'ESCC 2013 avoir déjà reçu un diagnostic de trouble de l'humeur ou d'anxiété.

\section{Collecte et traitement des données}

Statistique Canada a recueilli les données pour l'EPMCC-THA de 2014 en deux vagues de cinq semaines chacune : la première en novembre et décembre 2013 et la deuxième en février et mars 2014. Les répondants sélectionnés ont été interviewés à partir de centres d'appel centralisés à l'aide une application d'entrevue téléphonique assistée par ordinateur.

Statistique Canada a eu recours à plusieurs moyens pour réduire au minimum les nonréponses. Des lettres de présentation ont été envoyées aux répondants ciblés expliquant le but de l'enquête avant le début de la période de collecte des données. Les enquêteurs ont reçu comme consigne de prendre toutes les mesures raisonnables pour obtenir des entrevues : lorsque le numéro de téléphone indiqué sur l'ESCC 2013 n'était plus celui du répondant, on a tenté de trouver le bon numéro et, lorsque des personnes refusaient tout d'abord de participer, on a tenté d'autres mesures, en particulier une lettre suivie d'un deuxième appel, pour les convaincre de l'importance de participer à l'enquête. Enfin, pour éliminer toute barrière linguistique, Statistique Canada était prêt à recruter au besoin des enquêteurs parlant de nombreuses langues. Cela ne s'est pas révélé nécessaire, toutes 
TABLEAU 2

Taille initiale de l'échantillon, nombre modélisé de cas admissibles et taux de réponse selon le sexe et le groupe d'âge dans l'EPMCC-THA de 2014

\begin{tabular}{|lcccccc|}
\multicolumn{1}{|c}{ Sexe } & $\begin{array}{c}\text { Groupe } \\
\text { d'âge (ans) }\end{array}$ & $\begin{array}{c}\text { Taille de } \\
\text { l'échantillon } \\
\text { choisi }\end{array}$ & $\begin{array}{c}\text { Nombre } \\
\text { modélisé de } \\
\text { cas admissibles }\end{array}$ & $\begin{array}{c}\text { Taux modélisé } \\
\text { de cas } \\
\text { admissibles }\end{array}$ & $\begin{array}{c}\text { Nombre de } \\
\text { répondants }\end{array}$ & $\begin{array}{c}\text { Taux de } \\
\text { réponse }\end{array}$ \\
\hline Femmes & 18 à 34 & 875 & 720 & $82,3 \%$ & 436 & $60,6 \%$ \\
\hline & 35 à 49 & 741 & 672 & $90,7 \%$ & 453 & $67,4 \%$ \\
\hline & 50 à 64 & 1361 & 1211 & $89,0 \%$ & 892 & $73,7 \%$ \\
\hline Total & 65 et plus & 990 & 765 & $77,3 \%$ & 559 & $73,1 \%$ \\
\hline Hommes & 18 à 34 & 399 & 306 & $76,6 \%$ & 175 & $57,3 \%$ \\
\hline & 35 à 49 & 425 & 350 & $82,4 \%$ & 226 & $64,5 \%$ \\
\hline & 50 à 64 & 659 & 554 & $84,1 \%$ & 391 & $70,6 \%$ \\
\hline & 65 et plus & 425 & 302 & $71,2 \%$ & 229 & $75,7 \%$ \\
\hline Total & & 1908 & 1513 & $79,3 \%$ & 1021 & $67,5 \%$ \\
\hline Ensemble & & 5875 & 4881 & $83,1 \%$ & 3361 & $68,9 \%$ \\
\hline
\end{tabular}

Abréviation : EPMCC-THA, Enquête sur les personnes ayant une maladie chronique au Canada - Composante des troubles de I'humeur et d'anxiété.

les entrevues ayant été menées en anglais $(80 \%)$ ou en français $(20 \%)^{36}$.

Entre avril et septembre 2014, Statistique Canada a traité, estimé et consigné les données. La vérification, le codage et la création de variables dérivées et groupées ont été réalisés au stade de l'entrevue (vérification et codage) ou à celui du traitement des données (création de variables dérivées et de variables groupées). Le poids attribué à chaque personne dans le fichier de données final peut être interprété comme le nombre de personnes dans la population représentées par le répondant. La pondération dans l'EPMCC-THA de 2014 a débuté avec le poids partagé au niveau individuel de l'ESCC 2013, car la base d'échantillonnage de l'EPMCC-THA de 2014 a été constituée de répondants de cette enquête. Une méthode de pondération en cinq étapes a ensuite été utilisée pour ajuster le poids de l'échantillon pour les exclusions, la sélection de l'échantillon, les taux de répondants admissibles, les non-réponses et l'autorisation de partager et jumeler les données.

Vu la complexité du plan d'échantillonnage de l'EPMCC-THA de 2014, calculer la variance à l'aide de formules simples était impossible et il a fallu utiliser une méthode de rééchantillonnage. La méthode bootstrap est une technique d'inférence basée sur le rééchantillonnage successif. La méthode bootstrap employée dans l'enquête utilise l'échantillon existant pour construire des échantillons synthétiques appelés " réplicats ». Ces réplicats servent à estimer la variance d'un paramètre. Statistique Canada a élaboré des poids bootstrap coordonnés pour l'EPMCC-THA de 2014 parce qu'elle dépend de l'échantillon de l'ESCC 2013. Les 500 réplicats du fichier bootstrap partagé de l'ESCC 2013 ont donc constitué le point de départ des poids bootstrap pour l'ЕРМССТНA de 2014. Chaque réplicat bootstrap a été ajusté au moyen des cinq étapes mentionnées précédemment. De plus amples renseignements à propos du processus de pondération sont disponibles dans le guide de l'utilisateur de l'ESCC $2013^{22}$.

Dans le cas des répondants ayant consenti au partage et au jumelage de leurs réponses, les données de l'EPMCC-THA de 2014 ont été jumelées à celles de l'ESCC 2013.

\section{Confidentialité et disponibilité des données}

Pour assurer la confidentialité des données concernant les répondants, tous les identificateurs personnels ont été retirés du fichier partagé-jumelé. Les données ont pu être utilisées à partir d'octobre 2014 et ont été mises à la disposition de l'ASPC, de Santé Canada et des ministères de la Santé provinciaux. Les chercheurs et les tiers peuvent accéder aux données de l'EPMCC-THA de 2014 par l'entremise des centres de données de recherche établis dans les universités par Statistique Canada.

\section{Éthique}

Statistique Canada a statué qu'il n'était pas nécessaire d'obtenir l'approbation d'un comité d'éthique pour administrer l'EPMCCTHA de 2014, car aucune donnée physique n'était recueillie. Aucun risque d'atteinte à la vie privée ou à la confidentialité, au sens de la Politique d'évaluation des facteurs relatifs à la vie privée, n'ayant été relevé, le statisticien en chef a autorisé la tenue de l'enquête.

La participation à l'EPMCC-THA de 2014 était totalement volontaire et les entrevues par personne interposée n'étaient pas autorisées. La Loi sur la statistique ${ }^{\dagger}$ interdit à Statistique Canada de divulguer tout renseignement receuilli permettant d'identifier un particulier, une entreprise ou une organisation, à moins que le répondant n'ait donné son consentement. Les partenaires de partage de données de Statistique Canada pour l'ESCC (l'ASPC, Santé Canada et les ministères de la Santé provinciaux et territoriaux) ont accès aux données aux termes de leurs ententes respectives de partage de données. Les fichiers de données ne renferment des renseignements que sur les répondants ayant accepté que leurs données soient communiquées aux partenaires de partage de données de Statistique Canada et que leurs réponses à l'EPMCC-THA de 2014 soient jumelées à celles de l'ESCC 2013. Les identificateurs personnels ont été retirés des dossiers partagés pour assurer la confidentialité des données. Les personnes qui utilisent ces fichiers doivent certifier dès le départ qu'elles ne diffuseront en aucun cas des renseignements susceptibles d'identifier un répondant à l'enquête.

\section{Qualité des données}

\section{Cas non admissibles}

Les cas non admissibles correspondent aux répondants contactés pour participer à l'enquête mais ne devant pas être inclus parce qu'ils ne répondent pas aux critères d'admissibilité.

Les répondants à l'EPMCC-THA de 2014 ont été classés en deux groupes : les cas résolus et les cas non résolus. Les cas non

${ }^{\dagger}$ Loi concernant la statistique du Canada. Accessible à l'adresse : http://laws-lois.justice.gc.ca/fra/lois/S-19/ 
résolus correspondent aux répondants n'ayant pas pu être contactés pendant la collecte de données, d'où une incertitude à les classer parmi les cas admissibles ou non admissibles. Les cas résolus correspondent aux répondants sélectionnés pour faire partie de l'échantillon, avec qui Statistique Canada est entré en contact et dont on a pu déterminer s'ils étaient admissibles ou non. Par exemple, les répondants ayant déclaré dans l'ESCC 2013 souffrir d'un trouble de l'humeur ou d'anxiété mais ayant affirmé n'avoir jamais présenté l'un de ces troubles lors de l'entrevue pour l'EPMCC-THA de 2014 ont été classés comme non admissibles.

Afin de réduire au minimum le nombre de cas non admissibles dans l'EPMCC-THA de 2014, deux questions de suivi ont été incluses à l'intention des répondants ayant déclaré ne pas souffrir actuellement d'un trouble de l'humeur ou d'anxiété diagnostiqué par un professionnel de la santé. Ces questions visaient à identifier les répondants ayant déjà reçu un diagnostic d'un de ces troubles mais n'en présentant plus les symptômes, ou bien étant parvenus à améliorer leur état au moyen de médicaments ou de changements dans leur mode de vie. Ces répondants ont été inclus dans l'enquête, ce qui a réduit le nombre de cas non admissibles.

Si on tient compte du nombre de cas non admissibles, le nombre total de personnes ayant déclaré présenter un trouble de l'humeur ou d'anxiété n'était pas le même dans l'ESCC 2013 et dans l'EPMCC-THA de 2014. L'ESCC 2013 a probablement comptabilisé des répondants ayant erronément déclaré présenter un trouble de l'humeur ou d'anxiété (faux positifs). À l'opposé, l'EPMCC-THA de 2014 a probablement exclu des répondants souffrant réellement d'un trouble de l'humeur ou d'anxiété mais ne l'ayant pas indiqué afin d'éviter de participer à l'enquête (faux négatifs). Les vérifications poussées réalisées au moyen du module Confirmation du diagnostic ont pu accroître la validité des données de l'EPMCC-THA de 2014 concernant le diagnostic, mais aucune étude empirique n'ait été menée pour le confirmer.

\section{Erreurs d'enquête}

On divise généralement les erreurs d'enquête en erreurs d'échantillonnage et en erreurs non dues à l'échantillonnage. Les erreurs d'échantillonnage sont dues au fait que les inférences sur la population générale sont basées sur des renseignements obtenus sur un échantillon de cette population seulement, alors que les erreurs non dues à l'échantillonnage sont des erreurs systématiques ou aléatoires variées non liées à l'échantillonnage ${ }^{22}$.

\section{Erreurs d'échantillonnage}

Après exclusion de certains répondants pour des raisons opérationnelles, tous les répondants à l'ESCC 2013 ayant déclaré souffrir d'un trouble de l'humeur ou d'anxiété ont été sélectionnés pour l'EPMCC-THA de 2014. L'EPMCC-THA de 2014 ne constitue cependant pas un recensement des personnes atteintes d'un trouble de l'humeur ou d'anxiété, étant donné que l'ESCC 2013 est une enquête par échantillonnage : des estimations quelque peu différentes auraient été obtenues si un recensement direct avait été réalisé par les mêmes moyens (même questionnaire, mêmes enquêteurs, mêmes méthodes de traitement, etc.).

\section{Erreurs non dues à l'échantillonnage}

Ces erreurs peuvent survenir à chaque étape ou presque d'une enquête et sont attribuables à des problèmes concernant la couverture, la non-réponse, la réponse et le traitement ${ }^{21}$. Les erreurs non dues à l'échantillonnage ne peuvent pas être mesurées ou atténuées en augmentant la taille de l'échantillon.

Les erreurs de couverture surviennent lorsque certains cas sont omis (sous-couverture), dupliqués ou erronément inclus (surcouverture). L'EPMCC-THA de 2014 a la même couverture que l'ESCC 2013 dans les 10 provinces. Vu le taux élevé de couverture de l'ESCC, il est peu probable qu'un biais significatif ait été introduit dans les données de l'EPMCC-THA de 2014.

Les erreurs de non-réponse résultent de réponses insuffisantes aux questions de l'enquête et vont de non-réponses partielles (absence de réponse à une ou plusieurs questions) à une non-réponse complète. Dans l'EPMCC-THA de 2014, les personnes ayant fourni des réponses partielles à une ou plusieurs questions devaient être exclues de l'enquête, mais étant donné que, en général, les répondants vont au bout du questionnaire une fois l'entrevue commencée, les nonréponses partielles sont rares. La nonréponse complète a lieu lorsque l'enquêteur est incapable d'entrer en contact avec le répondant ou lorsque le répondant refuse de participer à l'enquête. Pour réduire les risques que certains répondants ne puissent être suivis entre l'administration de l'ESCC 2013 et celle de l'EPMCC-THA de 2014, la collecte de données a été divisée en deux vagues. Les personnes dont les données ont été recueillies dans les six premiers mois de l'ESCC 2013 ont été contactées lors de la première vague (novembre/ décembre 2013), alors que celles dont les données ont été recueillies dans les six derniers mois de l'ESCC 2013 ont fait partie de la deuxième vague (février/mars 2014). L'utilisation de ces deux vagues a permis de réduire la période séparant l'ESCC 2013 de l'EPMCC-THA de 2014 à 14 mois maximum. L'effet des non-réponses complètes a été neutralisé en ajustant le poids des personnes ayant répondu à l'enquête en fonction de celles n'ayant pas répondu, ce qui a aussi permis de réduire au minimum le biais de non-réponse.

Les erreurs de réponse peuvent survenir lorsque les enquêteurs comprennent mal les instructions, lorsque les répondants font des erreurs en répondant aux questions ou lorsque les réponses sont mal saisies dans le questionnaire. Statistique Canada a adopté des mesures d'assurance de la qualité pour réduire au minimum ces types d'erreurs. Parmi ces mesures figurent le recours à des enquêteurs très compétents, une formation poussée des enquêteurs sur les procédures d'enquête et sur le questionnaire, l'observation d'entrevues pour s'assurer du respect des techniques et des procédures et pour détecter les problèmes de conception dans les questionnaires et une mauvaise compréhension des instructions et enfin l'utilisation de procédures visant à réduire au minimum les erreurs de saisie de données. Les erreurs de traitement et de tabulation ont également été réduites au minimum, au moyen de vérifications du codage et de la qualité pour s'assurer de la logique du traitement.

\section{Analyse des données}

Nous avons réalisé des analyses descriptives pour déterminer les caractéristiques sociodémographiques des répondants, à l'aide du logiciel SAS Enterprise Guide, version 5.1 (SAS Institute, Cary, Caroline du Nord, États-Unis). Pour tenir compte de la répartition de l'échantillon et de la conception de l'enquête, toutes les estimations ont été pondérées au moyen des poids d'enquête produits par Statistique Canada afin de refléter la distribution selon 
l'âge et le sexe de la population des ménages âgée de 18 ans et plus dans les 10 provinces. De plus, des estimations de la variance (intervalles de confiance à $95 \%$ et coefficients de variation) ont été réalisées à l'aide des poids bootstrap fournis avec les données ${ }^{37}$.

Les caractéristiques sociodémographiques intégrées à cette analyse sont :

- $\quad$ sexe (femmes, hommes);

- âge (18 à 34 ans, 35 à 49 ans, 50 à 64 ans, 65 ans et plus);

- état matrimonial (célibataire; marié(e) ou en union libre; veuf(ve), séparé(e), divorcé(e));

- niveau de scolarité (études secondaires non terminées, diplôme d'études secondaires sans études postsecondaires, études postsecondaires non terminées, diplôme d'études postsecondaires);

- quintiles de suffisance du revenu des ménages;

- immigrant (oui, non);

- temps passé au Canada depuis l'immigration (20 ans ou moins, plus de 20 ans);

- Autochtone (oui, non);

- milieu de vie (urbain, rural);

- région de résidence (provinces de l'Atlantique, Québec, Ontario, Prairies, Colombie-Britannique);

- type de trouble autodéclaré diagnostiqué par un professionnel de la santé (trouble de l'humeur seulement, trouble d'anxiété seulement, troubles de l'humeur et d'anxiété).

Le revenu a été mesuré à l'aide des quintiles ajustés de suffisance du revenu des ménages. Les répondants ont été divisés en quintiles selon le rapport entre leur revenu familial total et le seuil de faible revenu correspondant à la taille de leur ménage et de leur collectivité, calculé par Statistique Canada. Cette valeur fournit, pour chaque répondant, une mesure relative du revenu du ménage par rapport au revenu du ménage de tous les autres répondants ${ }^{22}$. Dans le cas des répondants dont l'information sur le revenu était manquante, Statistique Canada a eu recours à la méthode appelée " estimateur du plus proche voisin », laquelle modélise le revenu selon la structure familiale, les données sociodémographiques, certaines variables liées à la santé et le revenu selon les renseignements fiscaux regroupés. Le revenu a été imputé pour $23 \%$ des répondants à l'EPMCC-THA de 2014 n'ayant pas fourni d'estimation à la question sur le revenu du ménage $(14,7 \%$ ayant déclaré totalement l'échelle de revenu, 4,1 \% ayant déclaré partiellement l'échelle de revenu et 4,7\% n'ayant fourni aucune information sur le revenu).

\section{FIGURE 1}

Conception de l'échantillon de l'EPMCC-THA 2014

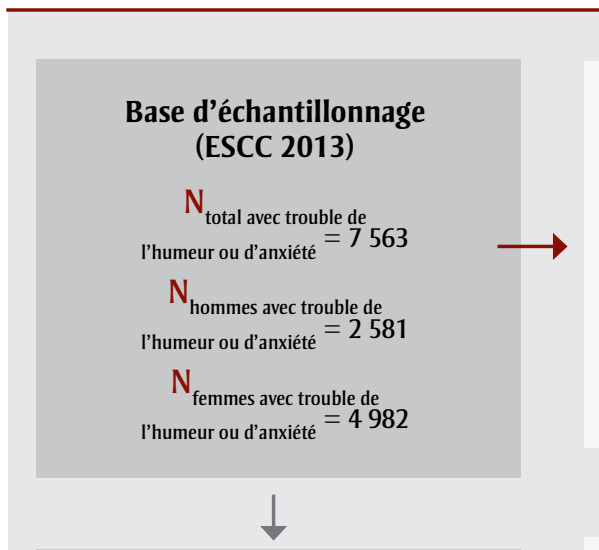

Échantillon sélectionné

$\mathrm{n}_{\text {total }}=5875$

$\mathrm{n}_{\text {hommes }}=1908$

$\mathrm{n}_{\text {femmes }}=3967$

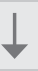

Échantillon admissible modélisé

$\mathrm{n}_{\text {total }}=4881$

$\mathrm{n}_{\text {hommes }}=1513$

$\mathrm{n}_{\text {femmes }}=3368$

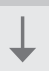

Échantillon final

(EMPCC-THA de 2014)

$$
\begin{gathered}
n_{\text {total }}=3361 \\
n_{\text {hommes }}=1021 \\
n_{\text {femmes }}=2340
\end{gathered}
$$

\section{Résultats}

Échantillon final de l'EPMCC-THA de 2014

La conception de l'échantillon de l'EPMCCTHA de 2014 est illustrée sur la figure 1 et la taille initiale de l'échantillon, les cas admissibles modélisés et le taux de réponse selon le sexe et le groupe d'âge sont présentés dans le tableau 2. Au total, 7563 répondants ayant déclaré souffrir d'un trouble de l'humeur ou d'anxiété diagnostiqué par un professionnel de la

\section{Exclusions prédéterminées}

$\mathrm{n}_{\text {exclusions }}=1688$

Critères d'exclusion :

- Sujets de moins de 18 ans

- Résidence dans l'un des trois territoires

- Ne pas avoir de numéro de téléphone valide

- Entrevue de l'ESCC par personne interposée

- Ne pas donner l'autorisation de partager les données ou de les jumeler à celles de l'ESCC

\section{Inadmissibles}

$n_{\text {inadmisibles }}=994$ (707 cas résolus et 287 estimés pour les cas non résolus)

Motifs d'inadmissibilité :

- Être incorrectement classé comme étant atteint d'un trouble dans l'ESCC

- Fournir délibérément des réponses afin d'être exclu de l'enquête

- Émigration

- Décès

\section{Non-réponse}

$\mathrm{n}_{\text {non-réponse }}=1520$

Motifs de non-réponse :

- Ne pas avoir de numéro de téléphone valide

- Ne pas accepter de participer

- Ne pas répondre à toutes les questions de l'enquête

- Ne pas donner l'autorisation de partager les données ou de les jumeler à celles de l'ESCC

Abréviations : EPMCC-THA, Enquête sur les personnes ayant une maladie chronique au Canada - Composante des troubles de l'humeur et d'anxiété; ESCC, Enquête sur la santé dans les collectivités canadiennes; $\mathrm{N}$, taille totale de la population; $\mathrm{n}$, taille du sous-échantillon. 
santé ont été inclus dans la base d'échantillonnage (ESCC 2013). Après application des critères d'exclusion prédéterminés pour l'EPMCC-THA de 2014, 1688 répondants ont été exclus.

Parmi les 5875 répondants sélectionnés pour l'EPMCC-THA de 2014, 707 se sont avérés non admissibles (cas résolus), ce qui s'est soldé par un taux de répondants non admissibles de 17,0 \%. De plus, 287 autres répondants ont été jugés non admissibles selon les prédictions du modèle logistique (cas non résolus).

Après exclusion des répondants non admissibles (cas résolus et cas non résolus), l'échantillon admissible modélisé comptait 4881 répondants (répondants admissibles à l'entrevue pour l'EPMCCTHA de 2014), ce qui représente un taux modélisé de répondants admissibles de 83,1\%.

Enfin, 1520 autres répondants ont été exclus en raison de non-réponses (complètes ou partielles), ce qui a donné un échantillon final pour analyse de 3361 répondants. Le taux de réponse global à l'EPMCC-THA de 2014 a donc été de $68,9 \%$. Les taux de réponse les plus bas ont été enregistrés parmi les hommes $(57,3 \%)$ et les femmes $(60,6 \%)$ de 18 à 34 ans.

\section{Caractéristiques sociodémographiques des}

Canadiens de 18 ans et plus avec troubles de l'humeur ou d'anxiété

Les caractéristiques sociodémographiques de la population avec troubles de l'humeur ou d'anxiété sont présentées dans les tableaux 3 et 4 et sur la figure 2. Près des deux tiers (64\%) étaient des femmes. La majorité était âgée de 50 à 64 ans, et les proportions de jeunes (18 à 34 ans) et d'âge moyen (35 à 64 ans) étaient assez également distribuées. Les aînés (65 ans et plus) ne représentaient que $15 \%$ de la population. Plus de la moitié (56 \%) étaient mariés ou vivaient en union libre, environ le quart étaient célibataires et les $18 \%$ restants étaient séparés, divorcés ou veufs. Près des deux tiers (60 \%) ont indiqué être titulaires d'un diplôme universitaire ou d'un certificat ou diplôme d'études postsecondaires. Le revenu des ménages était assez également distribué entre les quintiles de suffisance, avec cependant davantage d'individus ayant déclaré un revenu correspondant au quintile de suffisance le plus bas (23\%) que le plus élevé
TABLEAU 3

Caractéristiques sociodémographiques des Canadiens de 18 ans et plus atteints d'un trouble de l'humeur ou d'anxiété, EPMCC-THA de 2014

\begin{tabular}{|c|c|c|}
\hline \multirow[t]{2}{*}{ Caractéristiques sociodémographiques } & \multicolumn{2}{|c|}{$\begin{array}{l}\text { EPMCC-THA } 2014 \\
\qquad(n=3361)\end{array}$} \\
\hline & $\mathbf{n}^{\mathrm{a}}$ & \%b (IC à $95 \%)$ \\
\hline \multicolumn{3}{|l|}{ Sexe } \\
\hline Hommes & 1020 & $36,3(34,2$ à 38,4$)$ \\
\hline Femmes & 2341 & $63,7(61,6$ à 65,8) \\
\hline \multicolumn{3}{|l|}{ Âge (ans) } \\
\hline 18 à 34 & 610 & $26,0(24,0$ à 28,1$)$ \\
\hline 35 à 49 & 682 & $27,9(25,6$ à 30,3$)$ \\
\hline 50 à 64 & 1284 & 31,0 (28,9 à 33,2) \\
\hline 65 et plus & 785 & $15,0(13,8$ à 16,2$)$ \\
\hline \multicolumn{3}{|l|}{ État matrimonial } \\
\hline Célibataire/jamais marié(e) & 823 & $26,3(23,8$ à 28,7$)$ \\
\hline Séparé(e)/divorcé(e)/veuf(ve) & 926 & $17,7(15,6$ à 19,8$)$ \\
\hline Marié(e)/en union libre & 1610 & $56,1(53,2$ à 58,9$)$ \\
\hline \multicolumn{3}{|l|}{ Niveau de scolarité } \\
\hline Études secondaires non terminées & 528 & $12,7(10,9$ à 14,4$)$ \\
\hline Diplôme d'études secondaires & 728 & $21,5(19,1$ à 23,9$)$ \\
\hline Études postsecondaires non terminées & 176 & $5,6 \quad(4,3$ à 6,8$)$ \\
\hline $\begin{array}{l}\text { Certificat ou diplôme d'études postsecondaires, } \\
\text { ou diplôme universitaire }\end{array}$ & 1895 & $60,3(57,3$ à 63,2$)$ \\
\hline \multicolumn{3}{|l|}{ Quintiles de suffisance du revenu du ménage } \\
\hline Q1 (quintile inférieur) & 903 & $23,0(20,6$ à 25,3$)$ \\
\hline Q2 & 672 & $18,2(16,0$ à 20,4$)$ \\
\hline Q3 & 675 & $22,2(19,7$ à 24,7$)$ \\
\hline Q4 & 614 & $19,3(17,0$ à 21,6$)$ \\
\hline Q5 (quintile supérieur) & 497 & $17,3(15,0$ à 19,7$)$ \\
\hline \multicolumn{3}{|l|}{ Immigrant } \\
\hline Oui & 283 & $12,5 \quad(9,9$ à 15,1$)$ \\
\hline Non & 3074 & $87,5(84,9$ à 90,1$)$ \\
\hline \multicolumn{3}{|l|}{ Temps passé au Canada depuis l'immigration } \\
\hline 20 ans ou moins & 61 & $33,5(22,7$ à 44,3$)$ \\
\hline Plus de 20 ans & 222 & $66,5(55,7$ à 77,3$)$ \\
\hline \multicolumn{3}{|l|}{ Statut d'Autochtone } \\
\hline Oui & 201 & $5,1 \quad(3,8$ à 6,4$)$ \\
\hline Non & 2913 & $94,9(93,6$ à 96,2) \\
\hline \multicolumn{3}{|l|}{ Milieu de vie } \\
\hline Rural & 873 & $17,7(15,8$ à 19,6$)$ \\
\hline Urbain & 2488 & $82,3(80,4$ à 84,2$)$ \\
\hline \multicolumn{3}{|l|}{ Région } \\
\hline Atlantique & 508 & 8,9 (8,2 à 9,7) \\
\hline Québec & 593 & $20,3(18,6$ à 22,1$)$ \\
\hline Ontario & 1162 & $39,8(37,7$ à 42,0$)$ \\
\hline Prairies & 690 & $17,2(15,6$ à 18,8$)$ \\
\hline Colombie-Britannique & 408 & $13,7(12,0$ à 15,4$)$ \\
\hline
\end{tabular}

Suite page suivante 


\section{TABLEAU 3 (suite) \\ Caractéristiques sociodémographiques des Canadiens de 18 ans et plus atteints d'un trouble de l'humeur ou d'anxiété, EPMCC-THA 2014}

\begin{tabular}{|c|c|c|}
\hline \multirow[t]{2}{*}{ Caractéristiques sociodémographiques } & \multicolumn{2}{|c|}{$\begin{array}{l}\text { EPMCC-THA } 2014 \\
\qquad(n=3361)\end{array}$} \\
\hline & $\mathbf{n}^{\mathbf{a}}$ & \%b (IC à $95 \%)$ \\
\hline \multicolumn{3}{|l|}{ Type de trouble } \\
\hline Trouble de l'humeur seulement & 1531 & $45,2(42,2$ à 48,2$)$ \\
\hline Trouble d'anxiété seulement & 770 & $24,0(21,5$ à 26,6$)$ \\
\hline Trouble de l'humeur et trouble d'anxiété & 1060 & $30,7(28,0$ à 33,4$)$ \\
\hline
\end{tabular}

Abréviations : EPMCC-THA, Enquête sur les personnes ayant une maladie chronique au Canada - Composante des troubles de I'humeur et d'anxiété; IC, intervalle de confiance; Q, quintile.

${ }^{a}$ Nombres non pondérés.

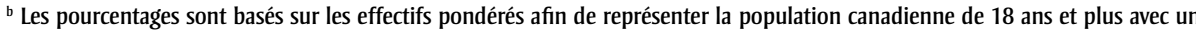
trouble de l'humeur ou d'anxiété et vivant dans les 10 provinces.

(17\%). La majorité (88\%) étaient nés au Canada, et les deux tiers (67\%) des immigrants vivaient au Canada depuis plus de 20 ans. La plupart (95 \%) étaient nonAutochtones, et la majorité (82\%) vivaient en milieu urbain. La répartition géographique de la population était la suivante : $40 \%$ en Ontario, $20 \%$ au Québec, $17 \%$ dans les Prairies, $14 \%$ en ColombieBritannique et $9 \%$ dans les provinces de l'Atlantique. Enfin, $45 \%$ ont déclaré avoir reçu un diagnostic d'un professionnel de la santé uniquement pour un trouble de l'humeur, $24 \%$ pour un trouble d'anxiété seulement et $31 \%$ pour les deux.

\section{Analyse}

L'EPMCC-THA de 2014 est la seule enquête populationnelle auprès des ménages qui, à ce jour, a fourni des données sur les Canadiens adultes aux prises avec un trouble de l'humeur ou d'anxiété diagnostiqué par un professionnel de la santé. Parmi les sujets abordés dans l'enquête figurent les activités quotidiennes et professionnelles, le stress, le sommeil, les relations sociales, les stratégies de gestion clinique (telles que les contacts avec des professionnels de la santé et les recommandations de ces derniers), la consommation de médicaments, les consultations psychologiques et l'autogestion. Cette enquête permet de combler d'importantes lacunes dans les données relevées par l'ASPC, la Commission de la santé mentale du Canada ${ }^{12}$ et d'autres organisations d'intervenants externes.

Nos résultats en ce qui concerne la distribution des caractéristiques sociodémographiques des Canadiens adultes vivant avec des troubles de l'humeur ou d'anxiété correspondent de manière générale à ceux d'autres études. Pour ce qui est du sexe, les troubles de l'humeur et d'anxiété se sont révélés constamment plus fréquents chez les femmes que chez les hommes ${ }^{38,39}$. De nombreux facteurs pourraient expliquer ce fait, qu'ils soient comportementaux (les femmes sont davantage portées que les hommes à tenter d'obtenir de l'aide) ${ }^{40}$, biologiques (les fluctuations hormonales liées à divers aspects de la fonction reproductrice prédisposeraient les femmes à la dépression) ${ }^{41}$ ou socioculturels (les femmes sont plus nombreuses que les hommes à faire état de stress lié à leurs responsabilités professionnelles et familiales) ${ }^{42}$.

Les études menées auprès de populations aux prises avec un trouble de l'humeur ou d'anxiété d'âge similaire (18 ans et plus) ont aussi montré que la distribution selon l'âge de ces troubles culmine à l'âge moyen et décline plus tard ${ }^{38,39,43}$. La plus forte proportion de répondants d'âge moyen pourrait être liée en partie aux difficultés particulières auxquelles sont souvent confrontées ces personnes, tel le stress qu'occasionne le déséquilibre entre le travail et la vie personnelle ou familiale $e^{44,45}$.

L'état matrimonial est un déterminant clé de la santé, et il est généralement admis que les personnes mariées sont en meilleure santé et présentent un risque moindre de mortalité que les personnes non mariées, en particulier les hommes ${ }^{46}$. Un peu plus de $50 \%$ des individus de notre étude étaient mariés ou vivaient en union libre, pourcentage un peu plus élevé que celui observé dans d'autres études canadiennes $^{38,39}$. Il est bon de souligner que ces autres études montraient que les personnes qui étaient atteintes d'un trouble de l'humeur ou d'anxiété étaient moins nombreuses à être mariées ou à vivre en union libre que celles qui ne l'étaient pas.

L'éducation est un autre important déterminant de la santé et peut avoir une influence

TABLEAU 4

Type de trouble de l'humeur ou d'anxiété selon le sexe et l'âge parmi les Canadiens de 18 ans et plus, EPMCC-THA de 2014

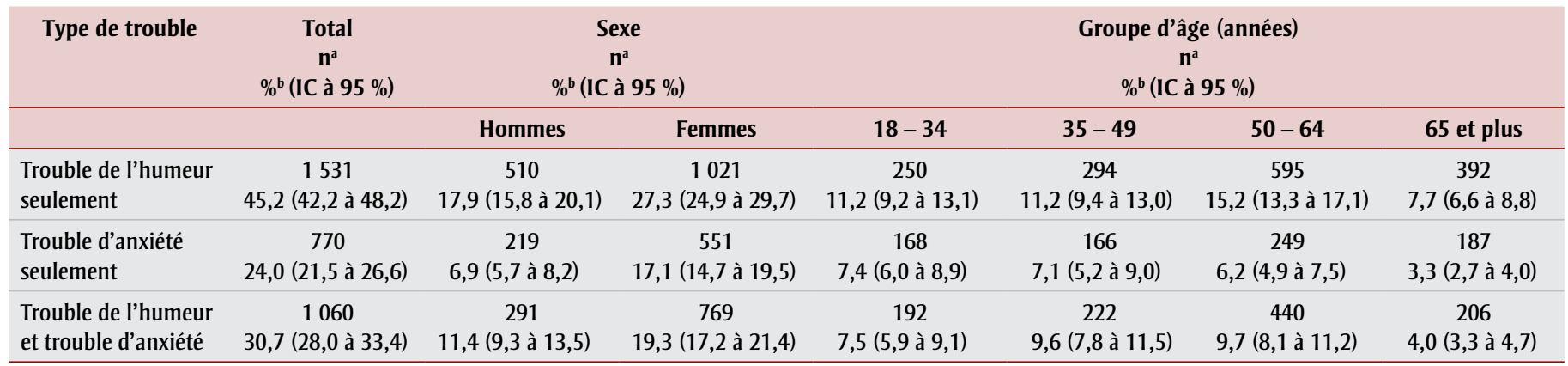

Abréviations : EPMCC-THA, Enquête sur les personnes ayant une maladie chronique au Canada - Composante des troubles de l'humeur et d'anxiété; IC, intervalle de confiance.

${ }^{a}$ Nombres non pondérés.

${ }^{\text {b }}$ Les pourcentages sont basés sur les effectifs pondérés afin de représenter la population canadienne de 18 ans et plus avec un trouble de l'humeur ou d'anxiété et vivant dans les 10 provinces. 
FIGURE 2

Distribution des Canadiens de 18 ans et plus avec un trouble de l'humeur ou d'anxiété selon a) l'état matrimonial, b) le niveau de scolarité, c) le quintile de suffisance du revenu du ménage, d) la région, e) le type de trouble selon le sexe et f) le type de trouble selon le groupe d'âge parmi les Canadiens de 18 ans et plus souffrant d'un trouble de l'humeur ou d'anxiété, EPMCC-THA de 2014
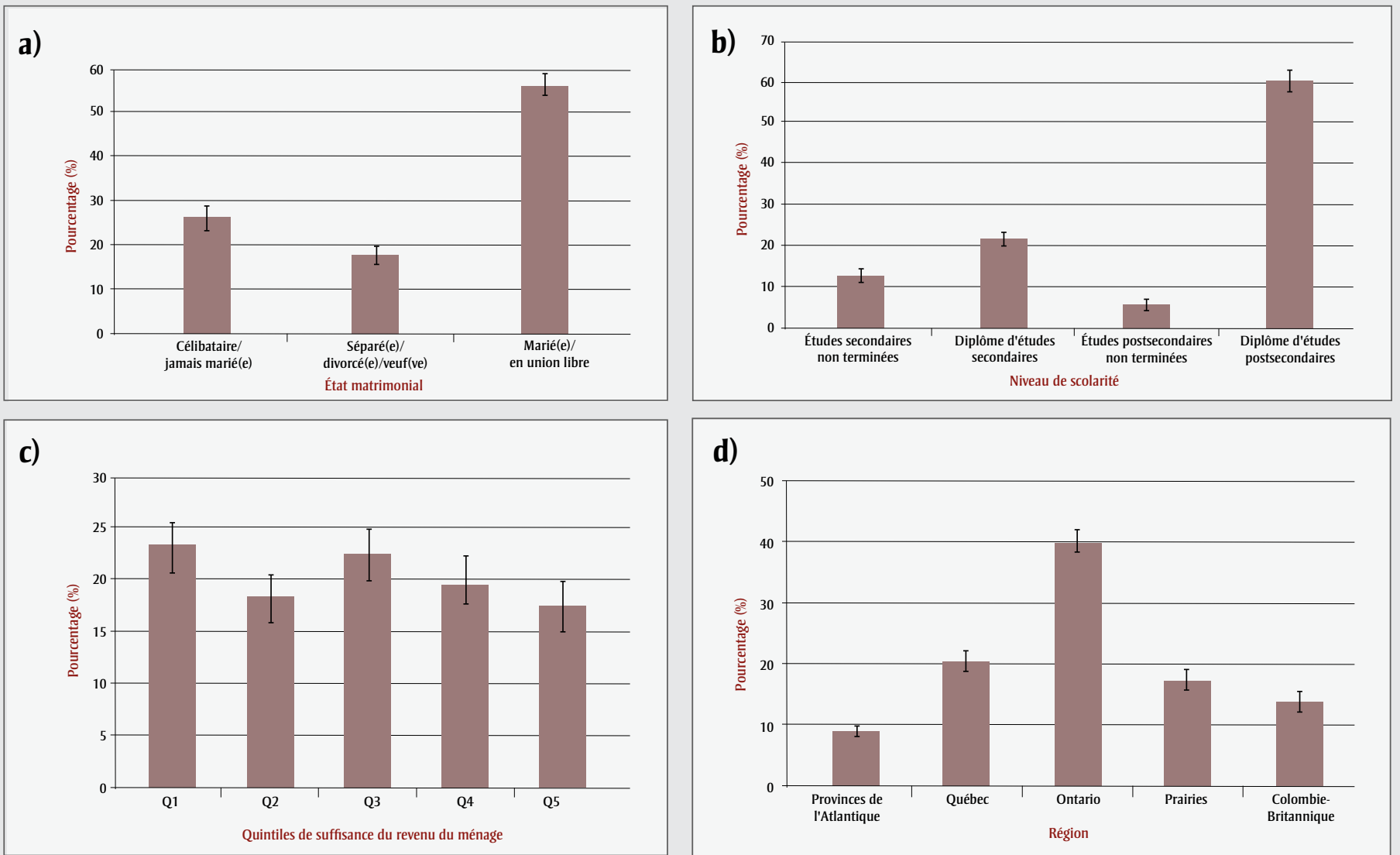

d)

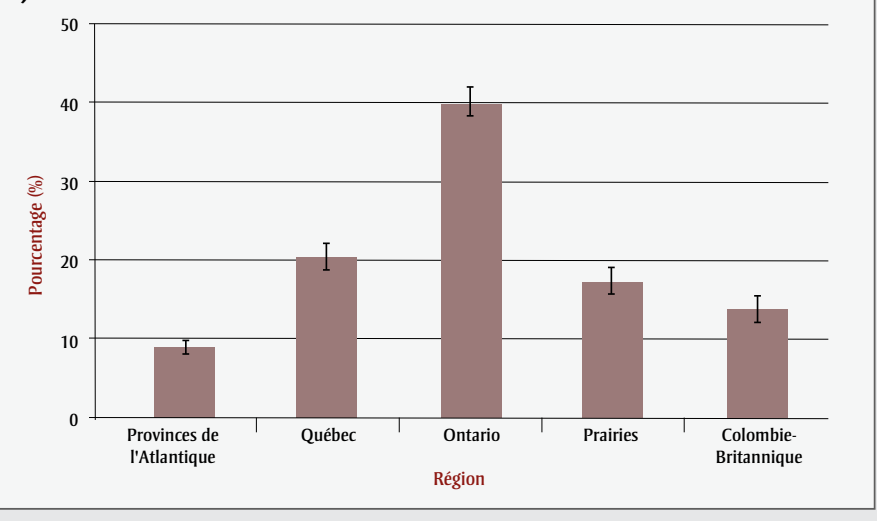

e)
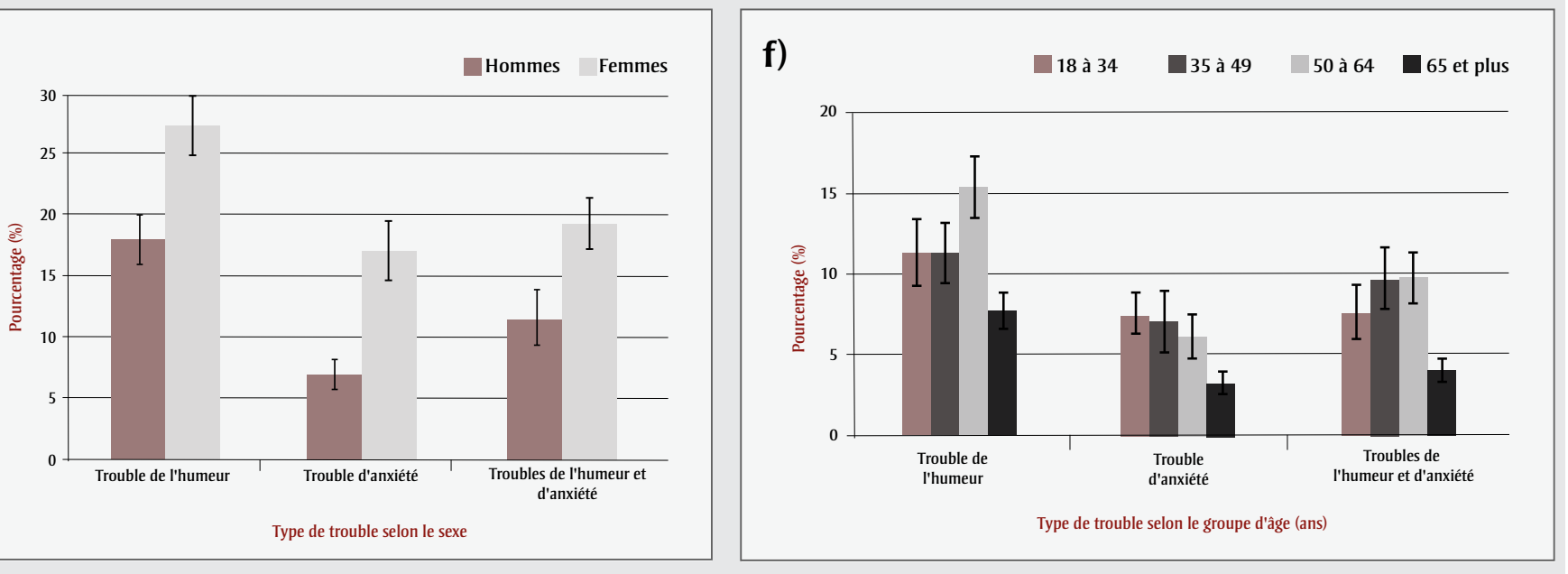

Abréviation : EPMCC-THA, Enquête sur les personnes ayant une maladie chronique au Canada - Composante des troubles de l'humeur et d'anxiété.

Remarques : Les proportions sont fondées sur les effectifs pondérés pour représenter la population canadienne de 18 ans et plus avec un trouble de l'humeur ou d'anxiété et vivant dans les 10 provinces. Les barres d'erreur représentent les intervalles de confiance à $95 \%$.

significative sur le niveau de revenu et sur le bienêtre économique ${ }^{47}$. La proportion de ceux ayant terminé des études postsecondaires était dans notre étude similaire à celle observée dans d'autres études canadiennes $^{38,39,48}$. Parmi ceux titulaires d'un diplôme d'études postsecondaires, dans toutes les études mentionnées plus haut, la proportion de personnes aux prises avec un trouble de l'humeur ou d'anxiété était similaire à celle des personnes qui ne souffraient pas de tels troubles ${ }^{38,39}$. Toutefois, après répartition selon le niveau d'études postsecondaires terminé, les personnes souffrant d'un trouble de l'humeur 
ou d'anxiété étaient moins nombreuses à avoir terminé leur baccalauréat ou des études supérieures que celles ne souffrant pas de tels troubles ${ }^{48}$. En ce qui concerne le revenu, davantage de personnes déclaraient un revenu du ménage se situant dans le plus bas quintile de suffisance du revenu que dans le quintile le plus, la suffisance de leur revenu étant généralement distribuée plus également dans les différents quintiles que dans les autres études ${ }^{38,39,48}$. Par conséquent, les résultats basés sur l'EPMCC-THA de 2014 relatifs à l'état de santé, aux répercussions des troubles sur les activités habituelles et professionnelles et aux stratégies de gestion adoptées pourraient représenter le scénario le plus favorable, étant donné la pléthore de données indiquant que les répondants ayant un statut socio-économique moindre sont en moins bonne santé et sont moins nombreux à adopter des comportements favorisant la santét ${ }^{4}$.

Les participants à notre étude étaient un peu plus nombreux en proportion à être immigrants que ceux d'autres études canadiennes $^{38,39}$. Dans ces autres études ${ }^{38,39}$, la proportion de personnes aux prises avec un trouble de l'humeur ou d'anxiété était plus faible parmi les immigrants, ce qui pourrait s'expliquer en partie par " l'effet de l'immigrant en bonne santé ", les personnes nées à l'étranger présentant un avantage sur le plan de la santé ${ }^{50}$. Sachant que cet effet s'amoindrit avec la durée de vie au Canada ${ }^{50}$, il est important de noter à ce propos qu'une grande proportion $(66,5 \%)$ des personnes ayant déclaré être immigrantes dans notre étude vivaient au Canada depuis plus de 20 ans.

Les proportions de personnes vivant en milieu rural et de personnes ayant déclaré être d'origine autochtone étaient comparables à celles des autres études menées au Canada $^{38,39}$. Ni ces études ni la nôtre n'ont démontré de différence en ce qui concerne la proportion de personnes atteintes de troubles de l'humeur et d'anxiété parmi celles vivant en milieu rural et parmi les Autochtones $^{38,39}$. Étant donné que le risque de maladie mentale est plus grand chez les Autochtones ${ }^{51,52}$ et que les personnes vivant dans les territoires, dans des réserves ou sur des terres publiques ont été exclues des autres études et de la nôtre, ces résultats pourraient être le reflet d'une sousreprésentation des Autochtones dans la population échantillonnée.
Enfin, le tiers des participants à notre étude ont déclaré avoir reçu à la fois un diagnostic de trouble de l'humeur et de trouble d'anxiété. Les études épidémiologiques menées en Europe et aux ÉtatsUnis font constamment état d'un taux élevé de comorbidité pour les troubles liés à la dépression et l'anxiété actuelles ou au cours de la vie, ce taux allant de $44 \%$ à $81 \%^{6-9}$. Les différences de base d'échantillonnage ou de critères diagnostiques pourraient expliquer en partie ces résultats différents entre études. Par ailleurs, on sait que les personnes avec divers problèmes de santé mentale concomitants vivent des répercussions significativement plus importantes sur leur santé et sur leur utilisation des services de santé mentale $e^{38,53,54}$. Étant donné que les différences entre les personnes présentant plus d'un trouble et celles n'en présentant qu'un pourraient être décelées en ce qui concerne l'état de santé des répondants, les répercussions de ces troubles sur les activités quotidiennes et professionnelles et les pratiques de gestion ou d'autogestion, stratifier les données de l'enquête par type de trouble serait utile.

\section{Points forts et limites}

L'EPMCC-THA de 2014 fournit des renseignements détaillés sur un vaste éventail de sujets liés aux expériences des Canadiens ayant un trouble de l'humeur ou d'anxiété, ce qui n'est pas possible dans une enquête sur la santé menée en population générale. De plus, les réponses ont été jumelées à celles de l'enquête source (l'ESCC 2013), ce qui a créé une série de données encore plus riche, étant donné que l'ESCC recueille aussi des renseignements liés à la santé sur par exemple les affections concomitantes, les comportements liés au mode de vie et les déterminants de la santé. On peut explorer les associations entre différents facteurs mais il est impossible d'en tirer des relations de cause à effet car il s'agit d'une enquête transversale ${ }^{55}$.

Bien que l'EPMCC-THA de 2014 ait été conçue de façon à être représentative à l'échelle nationale, la généralisation des données à l'ensemble de la population canadienne est limitée en raison de l'exclusion des personnes vivant dans les territoires et de certaines populations présentant un risque connu de maladie mentale, par exemple les Autochtones ${ }^{51,52}$ vivant dans des réserves ou sur des terres publiques, les sans-abri ${ }^{56}$, les patients vivant en établissement ${ }^{57}$ et les personnes emprisonnées ${ }^{58}$. L'échantillon est aussi limité aux Canadiens qui ont consulté un professionnel de la santé et ont obtenu un diagnostic de trouble de l'humeur ou d'anxiété. La possibilité de généraliser les résultats à ceux qui n'ont pas consulté un professionnel de la santé mais qui répondent tout de même aux critères diagnostiques du trouble de l'humeur ou d'anxiété est donc incertaine.

Comme c'est le cas pour la plupart des enquêtes populationnelles sur la santé, l'EPMCC-THA de 2014 s'appuie sur l'autodéclaration de troubles mentaux et de pratiques ou événements liés à la santé, qui n’ont été ni corroborés ni vérifiés par des tiers. Bien qu'il s'agisse de la méthode la plus pratique pour évaluer la maladie et ses répercussions dans de vastes études populationnelles, les événements autodéclarés liés à la santé pourraient être mal classés en raison du biais de désirabilité sociale, du biais de rappel ou d'une nondéclaration consciente, ce qui pourrait se solder par une sous-estimation ou une surestimation du fardeau de la maladie, tant à l'échelle individuelle qu'à celle de la société. Les recherches ont fait état d'une correspondance acceptable à bonne entre les affections physiques autodéclarées et les diagnostics posés par les professionnels de la santé ${ }^{59}$, mais, à notre connaissance, la validation des questions utilisées dans l'ESCC pour s'assurer de l'exactitude de l'autodéclaration des troubles de l'humeur ou d'anxiété n'a pas encore été faite.

Durant la période de collecte des données pour l'ESCC 2013 est paru le Manuel diagnostique et statistique des troubles mentaux, cinquième édition (DSM-V) ${ }^{60}$. Cependant, comme la collecte était déjà bien amorcée au moment de la transition entre le Manuel diagnostique et statistique des troubles mentaux, quatrième édition texte révisé (DSM-IV-TR) ${ }^{61}$ et le DSM-V, on trouve dans les questions de l'ESCC 2013 visant à déceler les cas les définitions des troubles de l'humeur ou d'anxiété présentées dans le DSM-IV-TR.

Des limites analytiques attribuables à la taille de l'échantillon sont à prévoir au moment de désagréger les données selon l'âge, le sexe (en particulier les hommes), la région ou d'autres caractéristiques importantes. Par exemple, il a été impossible de fournir une description statistique de la population par groupe ethnique, car les coefficients de variation (CV) des 
estimations pour les différentes catégories étaient élevés, ce qui indique une grande variabilité de l'échantillon et des estimations de qualité inacceptable. On a envisagé de regrouper les catégories Noirs, Arabes et Asiatiques dans une catégorie " personnes n'étant pas de race blanche ", mais cette mesure s'est soldée par une diversité du groupe de répondants, une variabilité persistante de l'échantillon $(\mathrm{CV}=22 \%)$ et une interprétation potentiellement limitée ou non significative des résultats.

Enfin, le taux de réponse à l'EPMCC-THA de 2014 (68,9 \%) était plus faible que dans les cycles précédents de l'EPMCC (75 \% à $83,2 \%)^{62,63}$. Les taux de réponse dans les enquêtes menées au Canada et ailleurs dans le monde connaissent de manière générale un déclin, et celui de l'EPMCCTHA de 2014 suit la tendance observée dans d'autres enquêtes générales ou axées sur la santét ${ }^{64}$. Étant donné que les nonrépondants n'ont pas consenti à ce que les données les concernant soient communiquées aux partenaires de partage de données de Statistique Canada, dont l'ASPC, leurs caractéristiques sociodémographiques et leurs raisons de ne pas participer à l'enquête sont inconnues.

\section{Conclusion}

Les troubles de l'humeur ou d'anxiété constituent les troubles mentaux les plus courants au Canada, et ils ont des répercussions majeures sur la vie quotidienne de ceux qui en souffrent ${ }^{1}$. Actuellement, l'EPMCCTHA de 2014, une enquête transversale de suivi de l'ESCC 2013, est la seule enquête populationnelle canadienne menée auprès des ménages fournissant des renseignements sur les répercussions de ces troubles sur les activités quotidiennes et professionnelles, ainsi que sur les stratégies de gestion. Les données de cette enquête peuvent aider les praticiens en santé publique et les cliniciens à déterminer les domaines où un soutien ou des interventions supplémentaires pourraient être utiles. Elles peuvent aussi fournir de l'information de base qui sera utile dans les futurs travaux de recherche en santé publique dans le domaine de la maladie mentale.

\section{Remerciements}

L'EPMCC-THA de 2014 a été financée par l'ASPC et conçue en collaboration avec Statistique Canada. L'élaboration de l'enquête a aussi été rendue possible grâce à la contribution des membres du Comité consultatif sur la surveillance de la santé mentale et de la maladie mentale de l'ASPC. L'ESCC de 2013 a été réalisée par Statistique Canada en partenariat avec Santé Canada et l'ASPC et a bénéficié d'un financement du gouvernement canadien. Aucun soutien financier ou matériel externe n'a été obtenu pour la tenue de cette étude.

\section{Conflits d'intérêts}

Les auteurs déclarent n'avoir aucun conflit d'intérêts.

\section{Références}

1. Pearson C, Janz T, Ali J. Troubles mentaux et troubles liés à l'utilisation de substances au Canada. Statistique Canada: Coup d'oeil sur la santé [Internet]. 18 septembre 2013 [mise à jour le 27 novembre 2015; consulté le 24 juin 2016]. En ligne à : http:// www.statcan.gc.ca/pub/82-624-x /2013001/article/11855-fra.htm

2. Agence de la santé publique du Canada. Les troubles anxieux et de l'humeur au Canada : points saillants de l'Enquête sur les personnes ayant une maladie chronique au Canada 2014 [Internet]. Ottawa (Ont.) : Gouvernement du Canada; 2015 [consulté le 24 juin 2016]. En ligne à : http://www.canadiensensante.gc.ca /publications/diseases-conditions -maladies-affections/mental-mood -anxiety-anxieux-humeur/index-fra .php

3. Gouvernement du Canada. Aspect humain de la santé mentale et de la maladie mentale au Canada 2006 Ottawa (Ont.) : Ministre des travaux publics et Services gouvernementaux Canada; 2006 [ $\mathrm{N}^{\circ}$ de catalogue HP5-19/2006F].

4. Devane CL, Chiao E, Franklin M, Kruep EJ. Anxiety disorders in the 21st century: status, challenges, opportunities, and comorbidity with depression. Am J Manag Care. 2005; 11(Suppl 12):S344-53.

5. Lenze EJ, Mulsant BH, Shear MK, et al. Comorbid anxiety disorders in depressed elderly patients. Am J Psychiatry. 2000;157(5):722-8.
6. Lamers F, van Oppen P, Comijs HC, et al. Comorbidity patterns of anxiety and depressive disorders in a large cohort study: the Netherlands Study of Depression and Anxiety (NESDA). J Clin Psychiatry. 2011 Mar;72(3):341-8.

7. de Graaf R, Bijl RV, Spijker J, Beekman AT, Vollebergh WA. Temporal sequencing of lifetime mood disorders in relation to comorbid anxiety and substance use disorders-findings from the Netherlands Mental Health Survey and Incidence Study. Soc Psychiatry Psychiatr Epidemiol. 2003 Jan;38(1): 1-11.

8. Brown TA, Campbell LA, Lehman CL, Grisham JR, Mancill RB. Current and lifetime comorbidity of the DSM-IV anxiety and mood disorders in a large clinical sample. J Abnorm Psychol. 2001 Nov;110(4):585-99.

9. Kessler RC, Berglund P, Demler O, et al. National Comorbidity Survey Replication. The epidemiology of major depressive disorder: results from the National Comorbidity Survey Replication (NCS-R). JAMA. 2003 Jun 18;289(23):3095-105.

10. Strine TW, Mokdad AH, Balluz LS, et al. Depression and anxiety in the United States: findings from the 2006 Behavioral Risk Factor Surveillance System. Psychiatr Serv 2008;59: 1383-1390.

11. Kessler RC, Adler L, Barkley R, et al. The prevalence and correlates of adult ADHD in the United States: results from the National Comorbidity Survey Replication. Am J Psychiatry 2006; 163(4):716-723.

12. Commission de la santé mentale du Canada. Changer les orientations, changer des vies : stratégie en matière de santé mentale pour le Canada. Calgary (AB) : Commission de la santé mentale du Canada; 2012.

13. Anderson IM, Haddad PM. CANMAT guidelines for depression: clear and user-friendly. J Affect Disord. 2009; 117(Suppl 1):S3-4.

14. Starkes JM, Poulin CC, Kisely SR. Unmet need for the treatment of depression in Atlantic Canada. Can J Psychiatry. 2005;50(10):580-90. 
15. Kasper S. Anxiety disorders: underdiagnosed and insufficiently treated. Int J Psychiatry Clin Pract. 2006;10 Suppl 1:3-9.

16. Kohn R, Saxena S, Levav I, Saraceno B. The treatment gap in mental health care. Bull World Health Organ. 2004;82(11):858-66.

17. Weisberg RB, Dyck I, Culpepper L, Keller MB. Psychiatric treatment in primary care patients with anxiety disorders: a comparison of care received from primary care providers and psychiatrists. Am J Psychiatry. 2007; 164(2):276-82.

18. Issakidis C, Andrews G. Service utilisation for anxiety in an Australian community sample. Soc Psychiatry Psychiatr Epidemiol. 2002;37(4):153-63.

19. Thompson A, Hunt C, Issakidis C. Why wait? Reasons for delay and prompts to seek help for mental health problems in an Australian clinical sample. Soc Psychiatry Psychiatr Epidemiol. 2004;39(10):810-817.

20. Statistique Canada. Enquête sur les personnes ayant une maladie chronique au Canada (EPMCC) - 2014 Composante des troubles d'humeur et d'anxiété [Internet]. Ottawa (Ont.) : Gouvernement du Canada; 2013 [consulté le 24 juin 2016]. En ligne à : http://www23.statcan.gc.ca/imdb /p2SV_f.pl?Function = getSurvey\&SDDS $=5160$

21. Statistique Canada. Enquête sur les personnes ayant une maladie chronique au Canada (EPMCC) : guide de l'utilisateur. Ottawa (Ont.) : Statistique Canada; 2014.

22. Statistique Canada. Enquête sur la santé dans les collectivités canadiennes - composante annuelle (ESCC) : guide de l'utilisateur 2013. Fichiers de microdonnées de l'enquête. Ottawa (Ont.) : Statistique Canada; 2014.

23. Statistique Canada. Enquête sur la santé dans les collectivités canadiennes - composante annuelle (ESCC) : questionnaire 2013. Ottawa (Ont.) : Statistique Canada; 2014.
24. Statistique Canada. Enquête sur les personnes ayant une maladie chronique au Canada (EPMCC) - composante de l'arthrite : questionnaire 2009. Ottawa (Ont.) : Statistique Canada; 2009.

25. Statistique Canada. Enquête sur les personnes ayant une maladie chronique au Canada (EPMCC) - composante sur les maladies respiratoires : questionnaire 2011. Ottawa (Ont.) : Statistique Canada; 2011.

26. Statistique Canada. Enquête sur les personnes ayant une maladie chronique au Canada (EPMCC) - composante sur le diabète : questionnaire 2011. Ottawa (Ont.) : Statistique Canada; 2011.

27. Statistique Canada. Enquête sur les personnes ayant une maladie chronique au Canada (EPMCC) - composante sur l'hypertension : questionnaire 2009. Ottawa (Ont.) : Statistique Canada; 2009.

28. Katzman MA, Bleau P, Blier P, et al. Canadian clinical practice guidelines for the management of anxiety, posttraumatic stress and obsessive-compulsive disorders. BMC Psychiatry. 2014;14(Suppl 1):S1. En ligne à : http://www.biomed central.com /content/pdf/1471-244X-14-S1-S1.pdf

29. Kennedy SH, Lam RW, Parikh SV, Patten SB, Ravindran AV. Canadian Network for Mood and Anxiety Treatments (CANMAT) clinical guidelines for the management of major depressive disorder in adults. J Affect Disord. 2009 Oct;117(Suppl 1): S1-S2. En ligne à : http://www.canmat.org /resources/CANMAT \% 20Depression \%20Guidelines \%202009.pdf

30. Adams PF, Kirzinger WK, Martinez ME. Summary health statistics for the U.S. population: National Health Interview Survey, 2012. Vital Health Stat 10. 2013;(259):1-95.

31. Cutrona CE, Russell DW. The provisions of social support and adaptation to stress. Advances Personal Relation. 1987;1:37-67.
32. Caron J. L'Échelle de provisions sociales : une validation québécoise. Sante Ment Que. 1996;21(2):158-180.

33. Caron J. Une validation de la forme abrégée de l'Échelle de provisions sociales : l'ÉPS-10 items. Sante Ment Que. 2013;38(1):297-318.

34. Collins, D. Pretesting survey instruments: an overview of cognitive methods. Qual Life Res. 2003;12(3): 229-238.

35. Presser S, Couper MP, Lessler JT, et al. Methods for testing and evaluating survey questions. Public Opin Q. 2004;68(1):109-130.

36. Statistique Canada. Enquête sur les personnes ayant une maladie chronique au Canada : dictionnaire de données. Ottawa (Ont.) : Statistique Canada; 2014.

37. Rust K, Rao JN. Variance estimation for complex surveys using replication techniques. Stats Methods Med Res. 1996;5:281-310.

38. Pelletier, L, O’Donnell, S, McRae, L, Grenier, J. Le fardeau économique de l'anxiété généralisée au Canada. Promotion de la santé et prévention des maladies chroniques au Canada. 2017; [sous presse].

39. Pelletier L, O’Donnell S, Dykxhoorn J, McRae L, Patten SB. Under-diagnosis of mood disorders in Canada. Epidemiol Psychiatr Sci. 2016 May 6:110. [Epub disponible avant la version imprimée.]

40. Kazanjian A, Morettin D, Cho R. Health care utilization by Canadian women. BMC Womens Health. 2004; 4(Suppl 1):S33.

41. Noble RE. Depression in women. Metabolism. 2005 May;54(5 Suppl 1):49-52 .

42. Duxbury L, Higgins C, Lee C. Workfamily conflict: a comparison by gender, family type, and perceived control. J Fam Issues. 1994 Sept;15(3): 449-66.

43. Hobbs MJ, Anderson TM, Slade T, Andrews G. Relationship between measurement invariance and agerelated differences in the prevalence of generalized anxiety disorder. J Affect Disord. 2014;152-154: 306-312. 
44. Siegrist J. Chronic psychosocial stress at work and risk of depression: evidence from prospective studies. Eur Arch Psychiatry Clin Neurosci. 2008 Nov;258 Suppl 5:115-9.

45. Wang JL, Lesage A, Schmitz N, Drapeau A. The relationship between work stress and mental disorders in men and women: findings from a population-based study. J Epidemiol Community Health. 2008 Jan;62(1): 42-7.

46. Robards J, Evandrou M, Falkingham J, Vlachantoni A. Marital status, health and mortality. Maturitas. 2012 Dec; 73(4):295-9. doi: 10.1016/j.maturitas .2012.08.007. [Epub 21 septembre 2012.]

47. Frank JW, Mustard JF. The determinants of health from a historical perspective. Daedalus. 1994;123(4):1-17.

48. Diotte, E. Santé mentale et égalité des droits : les troubles de l'humeur Analyse des données de l'Enquête sur la santé dans les collectivités canadiennes (ESCC) - Santé mentale de 2012. Ottawa (Ont.) : la Commission canadienne des droits de la personne; $2015\left[\mathrm{~N}^{\circ}\right.$ au catalogue : HR4-28 /2015F-PDF].

49. Pampel FC, Krueger PM, Denney JT. Socioeconomic disparities in health behaviors. Annu Rev Sociol. 2010 Aug;36:349-370.

50. Vang Z; Sigouin J, Flenon A, Gagnon A. The healthy immigrant effect in Canada: a systematic review. Population Change and Lifecourse Strategic Knowledge Cluster Discussion Paper Series. 2015;3(1):Article 4. En ligne à : http://ir.lib.uwo.ca/pclc/vol3/iss1/4

51. MacMillan HL, Jamieson E, Walsh CA, et al. First Nations women's mental health: results from an Ontario survey. Arch Women Ment Health. 2008;11(2): 109-15.

52. Gouvernement du Canada. Santé mentale et bien-être des populations autochtones au Canada. Dans le document : Aspect humain de la santé mentale et de la maladie mentale au Canada 2006. Ottawa (Ont.) : Gouvernement du Canada; 2006:15979 [ $\mathrm{N}^{\circ}$ de cat. HP5/19/2006F].
53. Kessler RC, McGonagle KA, Zhao S, et al. Lifetime and 12-month prevalence of DSM-III-R psychiatric disorders in the United States. Results from the National Comorbidity Survey. Arch Gen Psychiatry. 1994 Jan;51(1):8-19.

54. McLaughlin TP, Khandker RK, Kruzikas DT, Tummala R. Overlap of anxiety and depression in a managed care population: prevalence and association with resource utilization. J Clin Psychiatry. 2006 Aug;67(8):1187-93.

55. Bland M. An introduction to medical statistics. 3e éd. Oxford: Oxford University Press; 2001.

56. Krausz RM, Clarkson AF, Strehlau V, Torchalla I, Li K, Schuetz CG. Mental disorder, service use, and barriers to care among 500 homeless people in 3 different urban settings. Soc Psychiatry Psychiatr Epidemiol 2013 Aug;48(8): 1235-43.

57. Seitz D, Purandare N, Conn D. Prevalence of psychiatric disorders among older adults in long-term care homes: a systematic review. Int Psychogeriatr. 2010 Nov;22(7):1025-39.

58. Simpson AI, McMaster JJ, Cohen SN. Challenges for Canada in meeting the needs of persons with serious mental illness in prison. J Am Acad Psychiatry Law. 2013;41(4):501-9.

59. Kriegsman DM, Penninx BW, van Eijk JT, Boeke AJ, Deeg DJ. Self-reports and general practitioner information on the presence of chronic diseases in community dwelling elderly. A study on the accuracy of patients' selfreports and on determinants of inaccuracy. J Clin Epidemiol. 1996;49(12): 1407-17.

60. American Psychiatric Association. Diagnostic and statistical manual of mental disorders. 5e éd. Washington (DC): American Psychiatric Association; 2013.

61. American Psychiatric Association. Diagnostic and statistical manual of mental disorders. 4e éd., texte révisé. Washington, DC: American Psychiatric Association; 2000.
62. Statistique Canada. Enquête sur les personnes ayant une maladie chronique au Canada (EPMCC) - guide de l'utilisateur - décembre 2009 [Internet]. Statistique Canada; 2010 [consulté le 24 juin 2016]. En ligne à : http://www23.statcan.gc.ca/imdb -bmdi/pub/document/5160_D5 _T1_V1-fra.htm

63. Statistique Canada. Enquête sur les personnes ayant une maladie chronique au Canada (EPMCC) - guide de l'utilisateur - novembre 2011 [Internet]. Statistique Canada; 2011 [consulté le 24 juin 2016]. En ligne à : http:// www23.statcan.gc.ca/imdb-bmdi/pub /document/5160_D4_T1_V2-fra.htm

64. Bladon TL. The downward trend of survey response rates: implications and considerations for evaluators. Canadian J Prog Eval [Internet]. 2009 [consulté le 24 juin 2016]; 24(2):131156. En ligne à : http://www .evaluationcanada.ca/secure /24-2-131.pdf 